\section{Die Österreichische Apheresegesellschaft}

Online publiziert: 16. November 2013

(C) Springer-Verlag Wien 2013

Die Österreichische Apheresegesellschaft wurde von den Proponenten Univ.-Prof. Dr. Kurt Derfler und Univ.-Prof. Dr. Helmut Sinzinger am 25. Juli 2012 in Wien gegründet und am 10. April 2013 im Rahmen einer gemeinsamen wissenschaftlichen Sitzung in den Räumen der Gesellschaft der Ärzte in Wien vorgestellt.

Die Österreichische Apheresegesellschaft (Austrian Apheresis Society - AAA) bezweckt die Förderung, Unterstützung und Durchführung aller geeigneten Maßnahmen, um das Fachwissen und den wissenschaftlichen Fortschritt der Apheresetechniken in Diagnostik und Therapie fachlich geeignet $\mathrm{zu}$ vertreten und in der Öffentlichkeit bewusst zu machen.

Neben dem etablierten Verfahren der Lipoproteinapherese, das in Österreich seit mehr als 20 Jahren erfolgreich bei einer ständig steigenden Anzahl von PatientInnen mit schwerer Hypercholesterinämie angewandt wird, stellt diese neue Therapieoption nicht nur bei Fettstoffwechselstörungen, sondern auch bei Autoimmunerkrankungen, in der Transplantationsmedizin (Antikörper-mediierten Abstoßung) und anderen Indikationen eine wesentliche Zukunftshoffnung dar. Ein viel versprechender Ausblick für den Einsatz der Apherese-Technologie sind z. B. allergische Erkrankungen unter Anwendung therapeutischer Antikörper, die biotechnologisch in großen Mengen hergestellt die gezielte Entfernung von IgE-Antikörpern aus der Zirkulation ermöglichen.

Der derzeit amtierende Gründungsvorstand (Präsidium) der Gesellschaft besteht aus:Univ.-Prof. Dr. Helmut

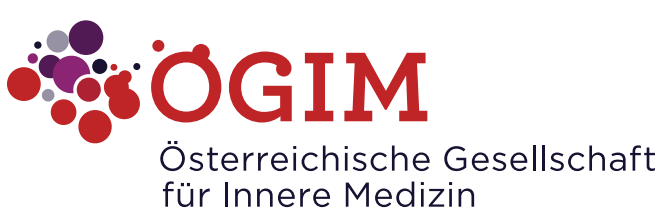

Sinzinger (Präsident), Univ.-Prof. Dr. Gert Mayer (Vizepräsident), Univ.-Prof. Dr. Kurt Derfler (Sekretär), Univ.Prof. Dr. Georg Stummvoll (Stellvertr. Sekretär), Doz. Dr. Sabine Steiner (Vermögensverwalterin), Univ.-Prof. Dr. Gerda Leitner (Stellvertr. Vermögensverwalterin).

Als erste wissenschaftliche Aktivität wurde ein Konsensus zur Lipoprotein-Apherese-Therapie verfasst (Titel: Lipoprotein-Apherese - Österreichischer Konsensus zu Indikation und Durchführung der Therapie. Autoren: Kurt Derfler, Sabine Steiner, Helmut Sinzinger - unter Mitarbeit von H. Drexel, A. Ferlitsch, M. Krainer, M. Clodi, R. Oberbauer, B. Paulweber, G. Mayer und H. Seyfried), der für die Indikation, Durchführung und Behandlung in Österreich einheitliche Standards setzen soll.

Ein geplantes österreichisches Aphereseregister soll einen besseren Überblick über die Behandlungssituation in Österreich geben. Eine Homepage der Vereinigung wird demnächst online zugänglich gemacht.

Die AAA ist in engem Kontakt mit der ISFA (International Society for Apheresis) bzw. WAA (World Apheresis Association), der europäischen bzw. weltweiten Fachorganisation.

Vereinsanschrift: 1090 Wien, Nadlergasse 1

Weitere Informationen: Prof. Dr. Helmut Sinzinger, E-Mail: helmut.sinzinger@meduniwien.ac.at 